\title{
A path integral ground state replica trick approach for the computation of entanglement entropy of dipolar linear rotors
}

Cite as: J. Chem. Phys. 152, 184113 (2020); https://doi.org/10.1063/5.0004602

Submitted: 13 February 2020 . Accepted: 21 April 2020 . Published Online: 14 May 2020

Tapas Sahoo (D), Dmitri louchtchenko (D), C. M. Herdman, and Pierre-Nicholas Roy (DD

This article may be downloaded for personal use only. Any other use requires prior permission of the author and AIP Publishing. This article appeared in J. Chem. Phys. 152, 184113 (2020) and may be found at https://doi.org/10.1063/5.0004602.

\section{Lock-in Amplifiers up to $600 \mathrm{MHz}$}
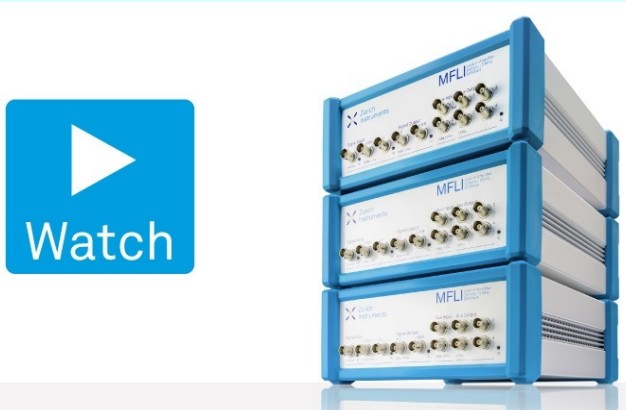


\title{
A path integral ground state replica trick approach for the computation of entanglement entropy of dipolar linear rotors
}

\author{
Cite as: J. Chem. Phys. 152, 184113 (2020); doi: 10.1063/5.0004602 \\ Submitted: 13 February 2020 - Accepted: 21 April 2020 • \\ Published Online: 14 May 2020
}

Tapas Sahoo,' (D) Dmitri louchtchenko, 'D C. M. Herdman, ${ }^{1,2}$ and Pierre-Nicholas Roy, ${ }^{1, a)}$ (D)

AFFILIATIONS

${ }^{1}$ Department of Chemistry, University of Waterloo, Waterloo, Ontario N2L 3G1, Canada

²Department of Physics, Middlebury College, Middlebury, Vermont 05753, USA

a) Author to whom correspondence should be addressed: pnroy@uwaterloo.ca

\begin{abstract}
We calculate the second Rényi entanglement entropy for systems of interacting linear rotors in their ground state as a measure of entanglement for continuous rotational degrees of freedom. The entropy is defined in relation to the purity of a subsystem in a bipartite quantum system, and to compute it, we compare two sampling ensembles based on the path integral ground state (PIGS) formalism. This scheme centers on the replica trick and is aided by the ratio trick, both developed in this context by Hastings et al. [Phys. Rev. Lett. 104, 157201 (2010)]. We study a system composed of linear quantum rotors on a lattice in one dimension, interacting via an anisotropic dipole-dipole potential. The ground state second Rényi entropies estimated by PIGS are benchmarked against those from the density matrix renormalization group for various interaction strengths and system sizes. We find that the entropy grows with an increase in interaction strength, and for large enough systems, it appears to plateau near $\log (2)$. We posit that the limiting case of many strongly interacting rotors behaves akin to a lattice of two-level particles in a cat state, in which one naturally finds an entanglement entropy of $\log (2)$.
\end{abstract}

Published under license by AIP Publishing. https://doi.org/10.1063/5.0004602

\section{INTRODUCTION}

Superposition and entanglement of quantum states are fundamental aspects of quantum information science and technologies such as quantum computing and quantum cryptography. Entanglement is present when the state of a many-body system cannot be factored into a product of individual one-body states (particle entanglement) or regions of space (spatial entanglement). A common measure of entanglement is von Neumann entropy $S_{\mathrm{vN}}\left(\hat{\varrho}_{A}\right)=-\operatorname{Tr}\left(\hat{\varrho}_{A} \log \hat{\varrho}_{A}\right)$, ${ }^{1}$ where $\hat{\varrho}_{A}$ is the reduced density matrix of a subsystem. $S_{\mathrm{vN}}\left(\hat{\varrho}_{A}\right)$ is a specific case of a family of entropies called Rényi entropies ${ }^{2}$ defined as $S_{n}\left(\hat{\varrho}_{A}\right)=\frac{1}{1-n} \log \left(\operatorname{Tr} \hat{\varrho}_{A}^{n}\right)$. The parameter $n$ is a non-negative real number, and $S_{\mathrm{vN}}\left(\hat{\varrho}_{A}\right)$ $=\lim _{n \rightarrow 1} S_{n}\left(\hat{\varrho}_{A}\right)$. Entanglement entropies have been extensively studied in theoretical physics to probe various properties of manybody quantum systems, particularly in condensed matter physics. For instance, the scaling behavior of the entanglement entropy allows one to distinguish phases that cannot be characterized based on symmetry properties alone, such as topological states of matter and spin liquids.

Hastings et al. have developed a quantum Monte Carlo procedure $^{6,7}$ to estimate the second Rényi entropy $S_{2}$, which they have applied to spin-1/2 Heisenberg models in one and two dimensions. The ground state entanglement entropy is estimated by computing the expectation value of the so-called SWAP operator acting on two replicas of the system. Humeniuk and Roscilde ${ }^{8}$ carried out finite temperature path integral Monte Carlo calculations of $S_{2}$ for planar rotors using an extended sampling ensemble. The computation of Rényi entropies has also been used to study entanglement in the continuum, ${ }^{9-12}$ particularly for translational motion in Cartesian coordinates.

In the present work, we examine a one-dimensional lattice of linear rotors with permanent dipoles interacting via an anisotropic dipole-dipole potential. To estimate $S_{2}$, we employ the "broken path" and "extended" ensembles to compute the purity $\operatorname{Tr} \hat{\varrho}_{A}^{2}$ of a reduced quantum state $\hat{\varrho}_{A}$ using path integral ground state 
(PIGS) $^{13,14}$ simulations based on the replica algorithm. Note that it is possible to use exact diagonalization and basis truncation techniques to compute the eigenstates of systems containing $\sim 10$ rotors, ${ }^{15}$ but that approach still suffers from exponential scaling when the system size increases. For larger systems, a density matrix renormalization group (DMRG) approach to compute the ground state of up to 100 rotors was recently developed and will be used here to benchmark our results. ${ }^{1}$

This article is organized as follows: We present the theoretical framework and provide details of the PIGS approach for linear rotors in Sec. II. The results are shown in Sec. III for a model Hamiltonian that represents a one-dimensional lattice of linear quantum rotors with anisotropic dipole-dipole interactions. We provide concluding remarks in Sec. IV.

\section{THEORETICAL FRAMEWORK}

Path integral Monte Carlo techniques have proven to be very effective for the simulation of many-body quantum systems. ${ }^{13}$ To study systems at zero temperature, the PIGS approach ${ }^{14}$ is highly efficient and can provide unbiased estimates of ground state observables. Below, we briefly review the PIGS approach in the context of linear rotors and show how one can compute $S_{2}$.

\section{A. Path integral ground state}

We consider systems of $N$ non-relativistic linear quantum rotors described by a time-independent Hamiltonian $\hat{H}=\hat{K}+\hat{V}$, where

$$
\hat{K}=\frac{B}{\hbar^{2}} \sum_{j=1}^{N} \hat{l}_{j}^{2}
$$

is the rotational kinetic energy (with a rotational constant $B$ ) and $\hat{V}$ is the interaction potential (which is diagonal in the position representation). As only rotational degrees of freedom (DOFs) are present, the position vector of the $j$ th rotor is denoted by polar and azimuthal angles, $\omega_{j}=\left(\theta_{j}, \phi_{j}\right)$. Moreover, we denote the position vectors of all the rotors collectively by $\boldsymbol{\Omega}=\left(\omega_{1}, \omega_{2}, \ldots, \omega_{N}\right)$.

Given a real-valued trial wavefunction $\Psi_{\mathrm{T}}(\Omega)$, one can obtain the ground state wavefunction $\Phi_{0}(\boldsymbol{\Omega})=\left\langle\Omega \mid \Phi_{0}\right\rangle$ for continuous angular motions of $N$ linear rotors in the position representation as

$$
\Phi_{0}(\boldsymbol{\Omega}) \propto \lim _{\beta \rightarrow \infty} \int d \boldsymbol{\Omega}^{\prime}\left\langle\boldsymbol{\Omega}\left|e^{-\frac{\beta}{2} \hat{H}}\right| \boldsymbol{\Omega}^{\prime}\right\rangle \Psi_{\mathrm{T}}\left(\mathbf{\Omega}^{\prime}\right) .
$$

The imaginary time projection operator $e^{-\frac{\beta}{2} \hat{H}}$ relaxes the trial wavefunction to the ground state in the $\beta \rightarrow \infty$ limit. The convergence rate of the limit in Eq. (2) is influenced by the choice of trial wavefunction, so one must choose it appropriately; in particular, there must be a non-zero overlap between the ground state and the trial wavefunction. For simplicity, we only use $\Psi_{\mathrm{T}}(\boldsymbol{\Omega})=1$ in the calculations below, which corresponds to a set of non-interacting rotors in their ground rotational states.

The ground state expectation value of an observable represented by the operator $\hat{\mathcal{O}}$ can be written in terms of the projected trial wavefunction as

$$
\langle\hat{\mathcal{O}}\rangle=\frac{\int d \boldsymbol{\Omega} \int d \boldsymbol{\Omega}^{\prime} \Psi_{\mathrm{T}}(\boldsymbol{\Omega})\left\langle\boldsymbol{\Omega}\left|e^{-\frac{\beta}{2} \hat{H}} \hat{\mathcal{O}} e^{-\frac{\beta}{2} \hat{H}}\right| \boldsymbol{\Omega}^{\prime}\right\rangle \Psi_{\mathrm{T}}\left(\boldsymbol{\Omega}^{\prime}\right)}{Z_{0}(\beta)},
$$

where $Z_{0}$ is a normalizing pseudo-partition function of the form

$$
Z_{0}(\beta)=\int d \boldsymbol{\Omega} \int d \boldsymbol{\Omega}^{\prime} \Psi_{\mathrm{T}}(\boldsymbol{\Omega})\left\langle\boldsymbol{\Omega}\left|e^{-\beta \hat{H}}\right| \mathbf{\Omega}^{\prime}\right\rangle \Psi_{\mathrm{T}}\left(\mathbf{\Omega}^{\prime}\right) .
$$

If the operator $\hat{\mathcal{O}}$ is diagonal in the position representation, the matrix element that appears in Eq. (3) can be evaluated as

$$
\begin{aligned}
& \left\langle\boldsymbol{\Omega}\left|e^{-\frac{\beta}{2} \hat{H}} \hat{\mathcal{O}} e^{-\frac{\beta}{2} \hat{H}}\right| \boldsymbol{\Omega}^{\prime}\right\rangle \\
& \quad=\int d \boldsymbol{\Omega}^{\prime \prime}\left\langle\boldsymbol{\Omega}\left|e^{-\frac{\beta}{2} \hat{H}}\right| \boldsymbol{\Omega}^{\prime \prime}\right\rangle \mathcal{O}\left(\boldsymbol{\Omega}^{\prime \prime}\right)\left\langle\boldsymbol{\Omega}^{\prime \prime}\left|e^{-\frac{\beta}{2} \hat{H}}\right| \boldsymbol{\Omega}^{\prime}\right\rangle .
\end{aligned}
$$

Since the operators $\hat{K}$ and $\hat{V}$ do not commute, the exponential appearing in Eq. (4) is factored into $P-1$ discrete imaginary time slices. Using the Trotter factorization scheme ${ }^{17}$ and the resolution of identity operator, one can expand the pseudo-partition function $Z_{0}(\beta)$ in a convenient form as

$$
\begin{aligned}
Z_{0}(\beta)= & \lim _{\tau \rightarrow 0} \int d \boldsymbol{\Omega}_{1} \cdots \int d \boldsymbol{\Omega}_{P} \Psi_{\mathrm{T}}\left(\boldsymbol{\Omega}_{1}\right) \Psi_{\mathrm{T}}\left(\boldsymbol{\Omega}_{P}\right) \prod_{i=1}^{P-1} \\
& \times \exp \left(-\frac{\tau}{2} V\left(\boldsymbol{\Omega}_{i}\right)\right)\left\langle\boldsymbol{\Omega}_{i}|\exp (-\tau \hat{K})| \boldsymbol{\Omega}_{i+1}\right\rangle \\
& \times \exp \left(-\frac{\tau}{2} V\left(\boldsymbol{\Omega}_{i+1}\right)\right),
\end{aligned}
$$

with $\tau=\beta /(P-1)$. The high-temperature rotational density matrix (referred to as a "link") can be expressed in terms of individual quantum rotors,

$$
\begin{aligned}
\left\langle\boldsymbol{\Omega}_{i}|\exp (-\tau \hat{K})| \boldsymbol{\Omega}_{i+1}\right\rangle & =\prod_{j=1}^{N}\left\langle\omega_{i, j}\left|\exp \left(-\tau \hat{K}_{j}\right)\right| \omega_{i+1, j}\right\rangle \\
& =\prod_{j=1}^{N} \varrho\left(\omega_{i, j}, \omega_{i+1, j} ; \tau\right) .
\end{aligned}
$$

These links connect adjacent "beads" with coordinates $\boldsymbol{\Omega}_{i}$ to form the path integral paths.

The high-temperature rotational density matrix ${ }^{18}$ for the $j$ th linear rotor can be written as

$$
\varrho\left(\omega_{i, j}, \omega_{i+1, j} ; \tau\right)=\sum_{l=0}^{\infty} \frac{2 l+1}{4 \pi} e^{-\tau B l(l+1)} P_{l}\left(\cos \left(\gamma_{i, j}\right)\right),
$$

where

$$
\begin{aligned}
\cos \left(\gamma_{i, j}\right)= & \sin \left(\theta_{i, j}\right) \sin \left(\theta_{i+1, j}\right) \cos \left(\phi_{i+1, j}-\phi_{i, j}\right) \\
& +\cos \left(\theta_{i, j}\right) \cos \left(\theta_{i+1, j}\right),
\end{aligned}
$$

and $P_{l}(x)$ is a Legendre polynomial.

\section{B. Replica trick}

In the present study, we wish to calculate the second Rényi entropy,

$$
S_{2}\left(\hat{\varrho}_{A}\right)=-\log \left(\operatorname{Tr} \hat{\varrho}_{A}^{2}\right),
$$

where $\hat{\varrho}_{A}$ is the reduced density operator of subsystem $A$, defined by the partial trace $\operatorname{Tr}_{B} \hat{\varrho}$ over the DOFs of partition $B$. We consider only symmetric splittings of one-dimensional systems into $A$ and $B$, such as those shown in Fig. 1. We will estimate $S_{2}$ for continuous rotational DOFs using the replica trick, ${ }^{6}$ which involves multiple copies of the system. 


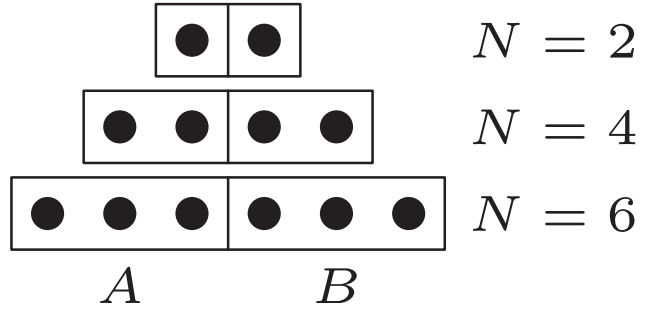

FIG. 1. Schematic representation of equal bipartitioning of 2,4 , and 6 rotors pinned on a linear lattice.

For a normalized pure state $\left|\Phi_{0}\right\rangle$, the density operator is $\hat{\varrho}=\left|\Phi_{0}\right\rangle\left\langle\Phi_{0}\right|$. The position representation of the density operator is the density matrix,

$$
\varrho\left(\boldsymbol{\Omega}_{A}, \boldsymbol{\Omega}_{B} ; \boldsymbol{\Omega}_{A}^{\prime}, \boldsymbol{\Omega}_{B}^{\prime}\right)=\left\langle\boldsymbol{\Omega}_{A} \boldsymbol{\Omega}_{B} \mid \Phi_{0}\right\rangle\left\langle\Phi_{0} \mid \boldsymbol{\Omega}_{A}^{\prime} \boldsymbol{\Omega}_{B}^{\prime}\right\rangle,
$$

where $\boldsymbol{\Omega}_{A}$ and $\boldsymbol{\Omega}_{B}$ collectively denote the position vectors of all the rotors in subsystems $A$ and $B$, respectively. One may readily write the partial trace in the position representation and calculate the reduced density matrix $\varrho_{A}\left(\boldsymbol{\Omega}_{A} ; \boldsymbol{\Omega}_{A}^{\prime}\right)$ for the subsystem $A$ by carrying out the integration over all DOFs of partition $B$,

$$
\varrho_{A}\left(\boldsymbol{\Omega}_{A} ; \boldsymbol{\Omega}_{A}^{\prime}\right)=\int d \boldsymbol{\Omega}_{B} \varrho\left(\boldsymbol{\Omega}_{A}, \boldsymbol{\Omega}_{B} ; \boldsymbol{\Omega}_{A}^{\prime}, \boldsymbol{\Omega}_{B}\right) .
$$

Squaring this gives us

$$
\begin{aligned}
\varrho_{A}^{2}\left(\boldsymbol{\Omega}_{A} ; \boldsymbol{\Omega}_{A}^{\prime \prime}\right)= & \int d \boldsymbol{\Omega}_{B} \int d \boldsymbol{\Omega}_{B}^{\prime} \int d \boldsymbol{\Omega}_{A}^{\prime}\left\langle\boldsymbol{\Omega}_{A} \boldsymbol{\Omega}_{B} \mid \Phi_{0}\right\rangle\left\langle\Phi_{0} \mid \boldsymbol{\Omega}_{A}^{\prime} \boldsymbol{\Omega}_{B}\right\rangle \\
& \times\left\langle\boldsymbol{\Omega}_{A}^{\prime} \boldsymbol{\Omega}_{B}^{\prime} \mid \Phi_{0}\right\rangle\left\langle\Phi_{0} \mid \boldsymbol{\Omega}_{A}^{\prime \prime} \boldsymbol{\Omega}_{B}^{\prime}\right\rangle,
\end{aligned}
$$

in which we find a second copy (or "replica") of the starting state and from which the purity may be obtained by taking the trace,

$$
\operatorname{Tr} \hat{\varrho}_{A}^{2}=\int d \boldsymbol{\Omega}_{A} \varrho_{A}^{2}\left(\boldsymbol{\Omega}_{A} ; \boldsymbol{\Omega}_{A}\right) .
$$

As described above, the projected state used in PIGS for a finite value of $\beta$ is

$$
\left|\Phi_{0}\right\rangle=\frac{e^{-\frac{\beta}{2} \hat{H}}\left|\Psi_{\mathrm{T}}\right\rangle}{\sqrt{Z_{0}(\beta)}},
$$

which leads to a factor of

$$
\begin{aligned}
Z_{0}^{2}(\beta)= & \int d \boldsymbol{\Omega}_{A} \int d \boldsymbol{\Omega}_{B} \int d \boldsymbol{\Omega}_{A}^{\prime} \int d \boldsymbol{\Omega}_{B}^{\prime} \\
& \times\left\langle\boldsymbol{\Omega}_{A} \boldsymbol{\Omega}_{B}\left|e^{-\frac{\beta}{2} \hat{H}}\right| \Psi_{\mathrm{T}}\right\rangle\left\langle\Psi_{\mathrm{T}}\left|e^{-\frac{\beta}{2} \hat{H}}\right| \boldsymbol{\Omega}_{A} \boldsymbol{\Omega}_{B}\right\rangle \\
& \times\left\langle\boldsymbol{\Omega}_{A}^{\prime} \boldsymbol{\Omega}_{B}^{\prime}\left|e^{-\frac{\beta}{2} \hat{H}}\right| \Psi_{\mathrm{T}}\right\rangle\left\langle\Psi_{\mathrm{T}}\left|e^{-\frac{\beta}{2} \hat{H}}\right| \boldsymbol{\Omega}_{A}^{\prime} \boldsymbol{\Omega}_{B}^{\prime}\right\rangle
\end{aligned}
$$

in the normalization of $\operatorname{Tr} \hat{\varrho}_{A}^{2}$. For brevity, we introduce the compact notation $\boldsymbol{\Gamma}=\left(\boldsymbol{\Omega}_{1}, \boldsymbol{\Omega}_{2}, \ldots, \boldsymbol{\Omega}_{P}, \boldsymbol{\Omega}_{1}^{\prime}, \boldsymbol{\Omega}_{2}^{\prime}, \ldots, \boldsymbol{\Omega}_{P}^{\prime}\right)$ for all the beads in both replicas and define the discretized path distribution

$$
\begin{aligned}
\Pi(\boldsymbol{\Gamma})= & \Psi_{\mathrm{T}}\left(\boldsymbol{\Omega}_{1}\right) \Psi_{\mathrm{T}}\left(\boldsymbol{\Omega}_{P}\right) \prod_{i=1}^{P-1} \exp \left(-\frac{\tau}{2} V\left(\boldsymbol{\Omega}_{i}\right)\right) \\
& \times\left\langle\boldsymbol{\Omega}_{i}|\exp (-\tau \hat{K})| \boldsymbol{\Omega}_{i+1}\right\rangle \exp \left(-\frac{\tau}{2} V\left(\boldsymbol{\Omega}_{i+1}\right)\right) \\
& \times \Psi_{\mathrm{T}}\left(\boldsymbol{\Omega}_{1}^{\prime}\right) \Psi_{\mathrm{T}}\left(\boldsymbol{\Omega}_{P}^{\prime}\right) \prod_{i=1}^{P-1} \exp \left(-\frac{\tau}{2} V\left(\boldsymbol{\Omega}_{i}^{\prime}\right)\right) \\
& \times\left\langle\boldsymbol{\Omega}_{i}^{\prime}|\exp (-\tau \hat{K})| \boldsymbol{\Omega}_{i+1}^{\prime}\right\rangle \exp \left(-\frac{\tau}{2} V\left(\boldsymbol{\Omega}_{i+1}^{\prime}\right)\right),
\end{aligned}
$$

composed of paths for both replicas. Hence, we may write Eq. (16) simply as

$$
Z_{0}^{2}(\beta)=\lim _{\tau \rightarrow 0} \int d \Gamma \Pi(\Gamma) .
$$

From this point onward, we will omit the $\tau$ limit, making each resulting expression into a finite- $P$ approximation.

The distribution $\Pi(\Gamma)$ is graphically depicted in Fig. 2, where the two independent replicas are clearly visible. After throwing away the extraneous pieces (namely, the beads prior to $M-1$ and after $M$ ), this graphical notation allows us to write

$$
Z_{0}^{2}(\beta)=\int d \boldsymbol{\Gamma}
$$

where we have chosen to draw only the relevant half of the interactions experienced by these beads. The purity can also be represented using the same diagrammatic notation,

$$
\operatorname{Tr} \hat{\varrho}_{A}^{2}
$$

$$
=\int d \boldsymbol{\Gamma}
$$
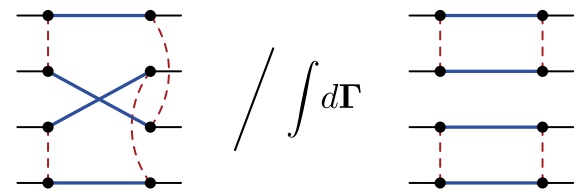

The integrand in the numerator has paths that are permuted between the two replicas at bead $M$. To compute this quantity, we evaluate the associated estimators over the configurations sampled by using a Monte Carlo algorithm for the two-replica system. We investigate below two ensembles and their corresponding estimators.

\section{Broken path ensemble}

We first describe the broken path distribution ${ }^{20}$

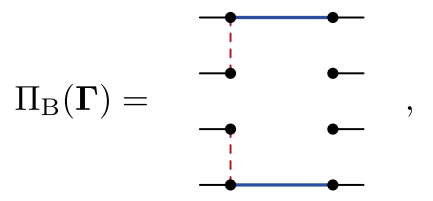




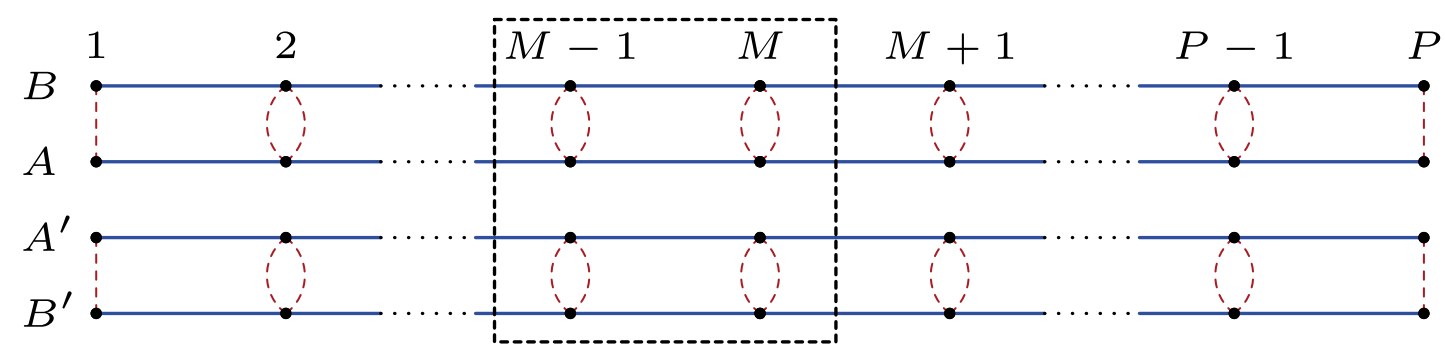

FIG. 2. Diagrammatic notation ${ }^{19,20}$ for $\Pi(\Gamma)$, showing the beads (points) connected by kinetic energy links (solid blue segments) and interactions (dashed red curves, each contributing only $V / 2$ ). As the middle bead $M$ and one adjacent bead (arbitrarily chosen to be $M-1$ ) play a crucial role in the computation of entanglement entropy, we highlight this region of interest with a dashed box. We identify all the links and beads of a single partition of a bipartite system by $A$ or $B$ in the diagram. The paths for two independent replicas of the system are represented as primed and unprimed.

along with the estimators

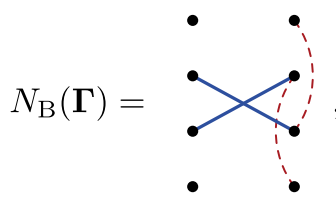

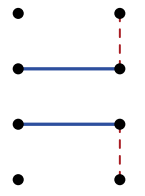

This distribution is identical to $\Pi(\Gamma)$, with the exception of removed links in partition $A$ between beads $M-1$ and $M$, causing a break in the path, and removed potential interactions between partitions $A$ and $B$ at bead $M$. That is,

$$
\Pi(\Gamma)=\Pi_{\mathrm{B}}(\boldsymbol{\Gamma}) D_{\mathrm{B}}(\boldsymbol{\Gamma}) .
$$

It is then clear that

$$
\operatorname{Tr} \hat{\varrho}_{A}^{2}=\frac{\int d \boldsymbol{\Gamma} \Pi_{\mathrm{B}}(\boldsymbol{\Gamma}) N_{\mathrm{B}}(\boldsymbol{\Gamma})}{\int d \boldsymbol{\Gamma} \Pi_{\mathrm{B}}(\boldsymbol{\Gamma})} / \frac{\int d \boldsymbol{\Gamma} \Pi_{\mathrm{B}}(\boldsymbol{\Gamma}) D_{\mathrm{B}}(\boldsymbol{\Gamma})}{\int d \boldsymbol{\Gamma} \Pi_{\mathrm{B}}(\boldsymbol{\Gamma})},
$$

so we may obtain the result via the following ratio of averages:

$$
\operatorname{Tr} \hat{\varrho}_{A}^{2}=\frac{\left\langle N_{B}\right\rangle}{\left\langle D_{B}\right\rangle},
$$

where the sampled configurations should be drawn from the broken path distribution.

\section{Extended ensemble}

Since the integrands of the numerator and denominator in Eq. (20) are, respectively, distributions of swapped and unswapped paths, we can consider these as independent configuration sectors of a larger ensemble. ${ }^{8}$ To do this, we apply the approach of Ref. 8 to PIGS and define

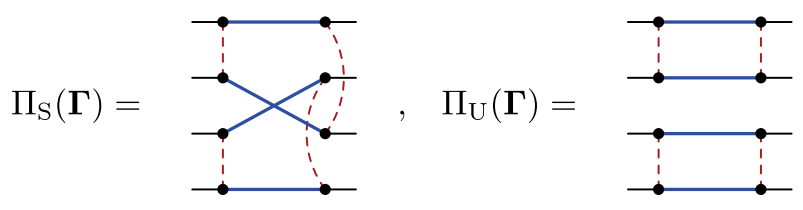

From these, we get the replicated pseudo-partition functions

$$
Z_{\mathrm{S}}=\int d \boldsymbol{\Gamma} \Pi_{\mathrm{S}}(\boldsymbol{\Gamma}), Z_{\mathrm{U}}=\int d \boldsymbol{\Gamma} \Pi_{\mathrm{U}}(\boldsymbol{\Gamma}),
$$

and

$$
Z_{\text {Total }}=\int d \Gamma\left(\Pi_{S}(\Gamma)+\Pi_{U}(\Gamma)\right)
$$

Thus,

$$
\operatorname{Tr} \hat{\varrho}_{A}^{2}=\frac{Z_{\mathrm{S}}}{Z_{\mathrm{U}}}=\frac{Z_{\mathrm{S}}}{Z_{\text {Total }}} / \frac{Z_{\mathrm{U}}}{Z_{\text {Total }}} .
$$

In the context of a Monte Carlo simulation, these sectors are akin to those found in a path integral worm calculation ${ }^{21}$ and require additional updates to change the distribution being sampled. Because the numbers of particles and beads are conserved between the sectors, these new updates are straightforward: after a batch of regular spatial updates, ${ }^{22}$ we propose changing to the connectivity of the beads, while the spatial configuration $\Gamma$ remains unchanged. If the distribution currently being sampled is $\Pi_{U}$, the proposed distribution is $\Pi_{S}$, and conversely, we propose $\Pi_{U}$ if the current distribution is $\Pi_{s}$. We then compute the standard Metropolis acceptance probability $^{23}$

$$
P\left(\Pi_{\mathrm{U}} \rightarrow \Pi_{\mathrm{S}}\right)=\min \left(1, \frac{\Pi_{\mathrm{S}}(\boldsymbol{\Gamma})}{\Pi_{\mathrm{U}}(\boldsymbol{\Gamma})}\right)
$$

[or its reciprocal analog $\left.P\left(\Pi_{\mathrm{S}} \rightarrow \Pi_{\mathrm{U}}\right)\right]$ and accept or reject the update accordingly.

The estimators necessary to evaluate Eq. (29) are extremely simple. Given that

$$
\begin{aligned}
\frac{Z_{S}}{Z_{\text {Total }}} & =\frac{\int d \boldsymbol{\Gamma}\left(1 \cdot \Pi_{S}(\boldsymbol{\Gamma})+0 \cdot \Pi_{\mathrm{U}}(\boldsymbol{\Gamma})\right)}{\int d \boldsymbol{\Gamma}\left(\Pi_{\mathrm{S}}(\boldsymbol{\Gamma})+\Pi_{\mathrm{U}}(\boldsymbol{\Gamma})\right)}, \\
\frac{Z_{\mathrm{U}}}{Z_{\text {Total }}} & =\frac{\int d \boldsymbol{\Gamma}\left(0 \cdot \Pi_{\mathrm{S}}(\boldsymbol{\Gamma})+1 \cdot \Pi_{\mathrm{U}}(\boldsymbol{\Gamma})\right)}{\int d \boldsymbol{\Gamma}\left(\Pi_{\mathrm{S}}(\boldsymbol{\Gamma})+\Pi_{\mathrm{U}}(\boldsymbol{\Gamma})\right)},
\end{aligned}
$$

all that needs to be evaluated after a simulation is the number $N_{S}$ of Monte Carlo steps that were spent in the swapped sector (and the complementary $N_{U}$ ). After this simple counting procedure, one only needs to find the quotient

$$
\operatorname{Tr} \hat{\varrho}_{A}^{2}=\frac{N_{S}}{N_{U}} .
$$




\section{Ratio trick}

For a system composed of $N$ rotors, the general form of the purity for a symmetric bipartition is given by

$$
\operatorname{Tr} \hat{\varrho}_{A}^{2}=\frac{Z^{(N / 2)}}{Z^{(0)}}
$$

where $Z^{(j)}$ is the replicated pseudo-partition function with $j$ paths swapped. In particular, $Z^{(N / 2)}=Z_{\mathrm{S}}$ and $Z^{(0)}=Z_{\mathrm{U}}$. Because the purity decays exponentially with $S_{2}$, this ratio becomes vanishingly small for strongly entangled systems and therefore difficult to compute using Monte Carlo.

This drawback can be circumvented by introducing the wellknown ratio trick ${ }^{6,7,9,24}$ where one expresses the purity as the product

$$
\operatorname{Tr} \hat{\varrho}_{A}^{2}=\prod_{j=1}^{N / 2} \frac{Z^{(j)}}{Z^{(j-1)}} .
$$

Although the number of ratios $\frac{Z^{(j)}}{Z^{(j-1)}}$ to be computed increases linearly with the system size, each ratio should be more amenable to Monte Carlo sampling.

Since, in the present work, we are only interested in ground states on a one-dimensional lattice, we do not expect the entanglement entropy to become very large. However, in higher dimensions, where the area law scaling of entanglement results in growth of entanglement entropy with the system size, the ratio trick may become necessary. Additionally, because each ratio in Eq. (35) may be computed independently, it enables us to run several instances of Monte Carlo sampling in parallel.

\section{NUMERICAL RESULTS}

We use the same Hamiltonian as the one previously used in PIGS studies of a linear chain of $O(3)$ dipolar rotors, ${ }^{25,26}$

$$
\hat{H}=\frac{B}{\hbar^{2}} \sum_{i=1}^{N} \hat{l}_{i}^{2}+\frac{\mu^{2}}{4 \pi \epsilon_{0} r^{3}} \sum_{i<j} \frac{\hat{x}_{i} \hat{x}_{j}+\hat{y}_{i} \hat{y}_{j}-2 \hat{z}_{i} \hat{z}_{j}}{|i-j|^{3}},
$$

where $\hat{l}_{i}^{2}$ is the squared angular momentum operator of the $i$ th rotor with the rotational constant $B$. The second term is the pairwise dipole-dipole interaction potential operator $\hat{V}$ for rotors placed along the spaced-fixed $z$ axis. The operators $\hat{x}_{i}, \hat{y}_{i}$, and $\hat{z}_{i}$ are the three unit vector components of the $i$ th rotor in the space-fixed $x$, $y$, and $z$ directions, and $\mu$ is its dipole moment. The lattice spacing is $r$, and $\epsilon_{0}$ is the permittivity of vacuum. In our calculations, $B=20.561 \mathrm{~cm}^{-1}$ and $r=10.05 \AA$. This choice of parameters is meant to mimic a linear chain of $\mathrm{HF}$ at $\mathrm{C}_{60}$ endofullerenes as studied in Ref. 15 and motivated by recent experimental ${ }^{27}$ and theoretical ${ }^{28}$ advances. As in Ref. 25, we define the interaction strength to be the dimensionless quantity

$$
g=\frac{\mu^{2}}{4 \pi \epsilon_{0} r^{3} B} .
$$

We first determine the convergence properties of $S_{2}$ with respect to the projection time $\beta$ and the imaginary time step $\tau$ for various system sizes $N$ and interaction strengths $g$. In the $\beta \rightarrow \infty$ limit, the trial wavefunction should relax to the exact ground state, while the systematic Trotter error can be eliminated by taking the $\tau \rightarrow 0$ limit. We express both $\beta$ and $\tau$ in reciprocal temperature units. We have implemented the sampling ensembles and estimators in our in-house software, MoRiBS-PIMC, a program to simulate molecular rotors in bosonic solvents using path-integral Monte Carlo. ${ }^{29}$ We refer the reader to Refs. 29 and 30 for further details regarding the simulation of molecular rotors in quantum environments.

Figure 3 shows $S_{2}$ as a function of $\beta$ estimated at a fixed value of $\tau=0.005 \mathrm{~K}^{-1}$ using the extended ensemble and the ratio trick. As expected, $S_{2}$ increases with an increase in $\beta$ and then reaches a plateau. This is because at $\beta=0$, the system is described by the trial wavefunction, which is a product state. As $\beta$ increases, more entanglement is built into the wavefunction until convergence is reached. Based on these results, we have chosen $\beta=0.2 \mathrm{~K}^{-1}$ as sufficient for convergence.
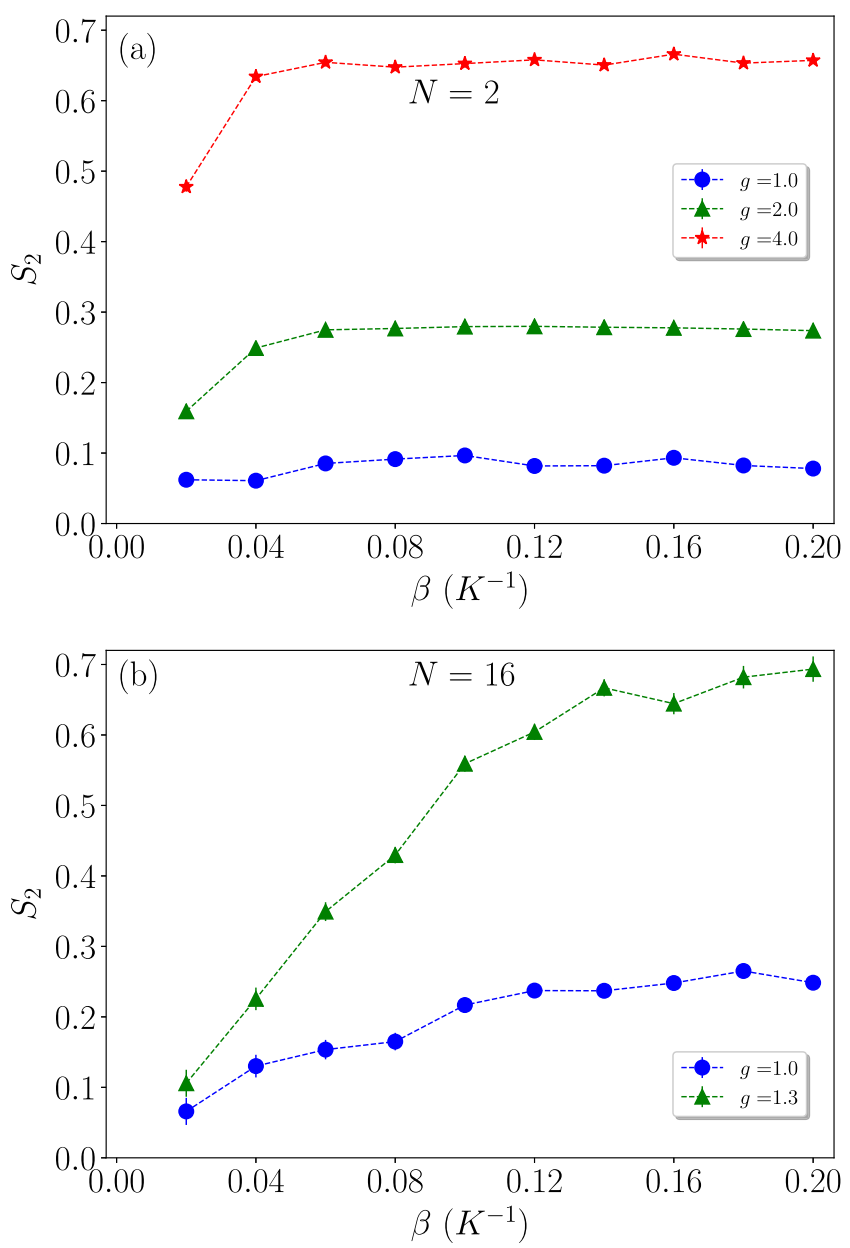

FIG. 3. Convergence of $S_{2}$ with an increase in $\beta$ for $N=2$ and $N=16$. When they are smaller than the symbols, statistical error bars are not visible. 
In order to remove the systematic error due to the Trotter factorization, we perform a series of simulations for varying $\tau$ values while maintaining $\beta$ fixed at $0.2 \mathrm{~K}^{-1}$. As demonstrated in the Appendix, we can then extrapolate the results to $\tau=0$ by fitting the estimated data to $S_{2}(\tau)=S_{2}^{\tau=0}+a \tau^{2}$, where $a$ is a fitting parameter. Calculated $S_{2}$ values for a series of $\tau$ points are shown in Figs. 4 and 5. The extrapolated values agree with the DMRG results obtained using the approach of Ref. 16.

To determine the importance of the ratio trick for this system, we have performed PIGS calculations to compute $S_{2}$ for $N=4,8,16$ using the extended ensemble. The total number of Monte Carlo samples in both cases (with or without the ratio trick) is the same. The results are compared to our benchmark DMRG data and are shown in Fig. 6. For the systems in question, we find no savings in total central processing unit (CPU) time when using the ratio trick, but there is a practical benefit in the wall time when using $N / 2$ shorter calculations as they are trivially parallelizable.
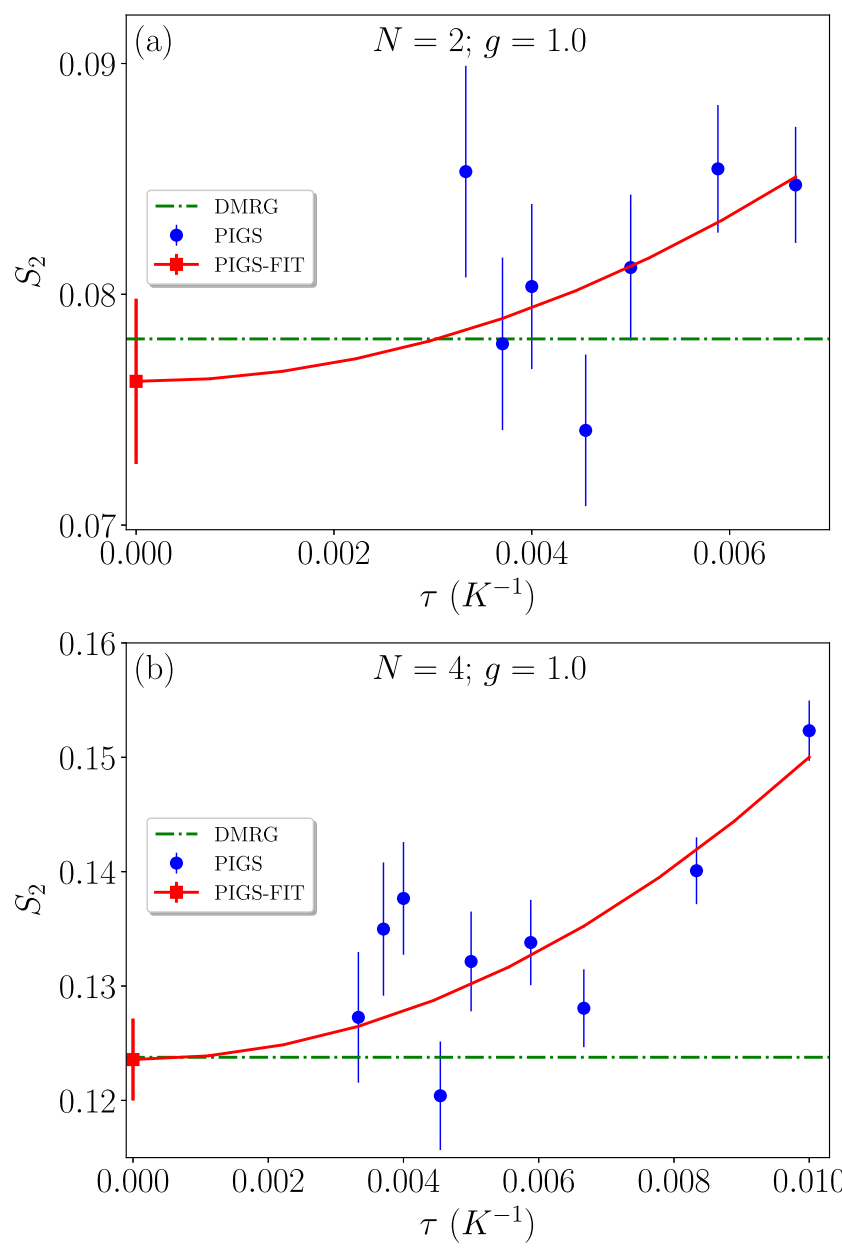

FIG. 4. Convergence of $S_{2}$ with a decrease in $\tau$ at $\beta=0.2 \mathrm{~K}^{-1}$ for $N=2$ and $N=4$. The red solid line represents a quadratic fit to the PIGS data, and the extrapolated $S_{2}$ value at $\tau \rightarrow 0$ is shown as a square marker.
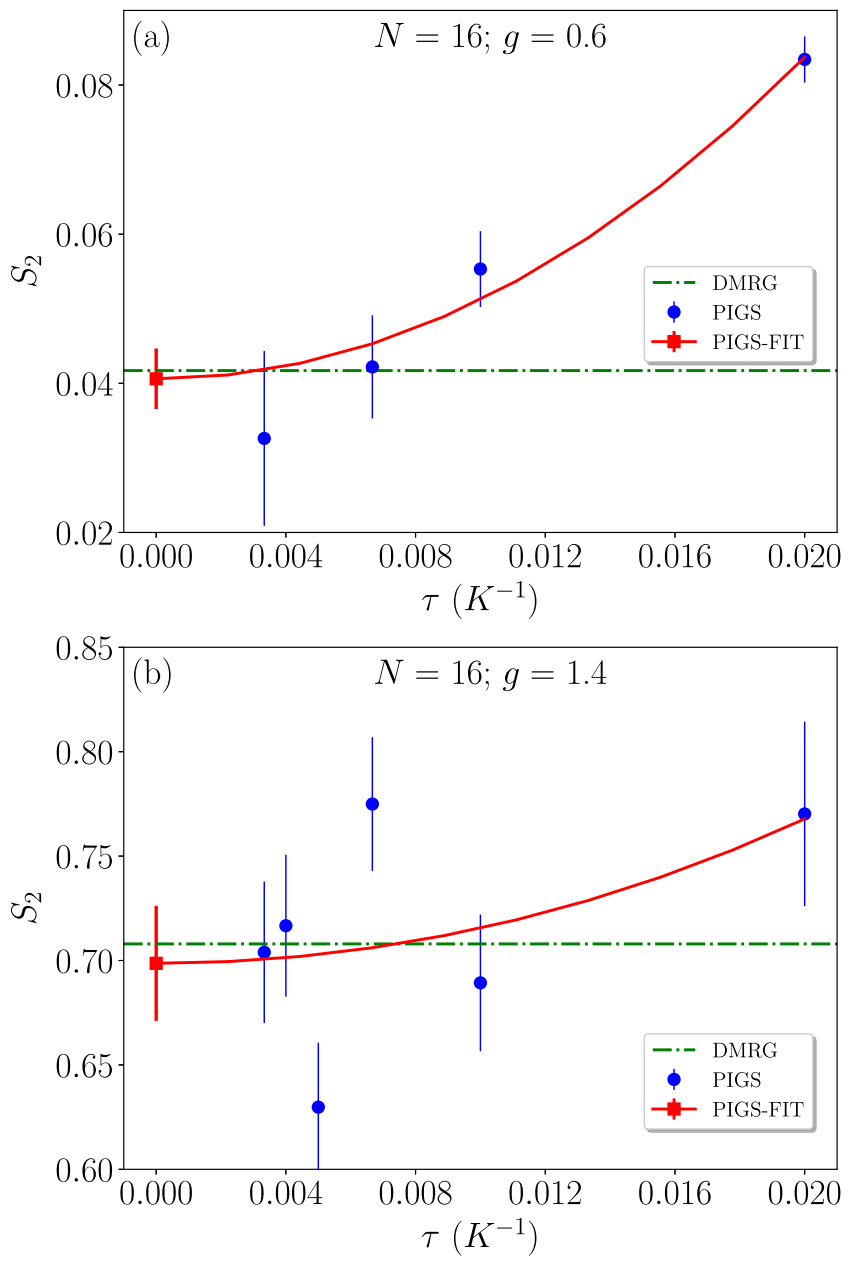

FIG. 5. Convergence of $S_{2}$ with a decrease in $\tau$ at $\beta=0.2 \mathrm{~K}^{-1}$ for $N=16$. The red solid line represents a quadratic fit to the PIGS data, and the extrapolated $S_{2}$ value at $\tau \rightarrow 0$ is shown as a square marker.

We also compare the broken path and extended ensembles in Fig. 7 for $N=2,4,8$. Neither performs noticeably better than the other, so we have chosen to use the extended ensemble solely on the grounds that the estimators are substantially simpler. While we do not find a computational advantage in using the ratio trick or extended ensemble in this one-dimensional system, in higher dimensional systems where there is a more significant growth of entanglement with the sub-system size, these methods may provide a distinct computational advantage.

Having compared the possible methods and performed the necessary convergence studies, we finally examine the behavior of $S_{2}$ over a range of $N$ and $g$ values. To do this, we use the extended ensemble along with the ratio trick to compute the subsystem purity. The results are shown in Fig. 8, where we observe good agreement between the PIGS estimates of $S_{2}$ and the DMRG benchmark results. 

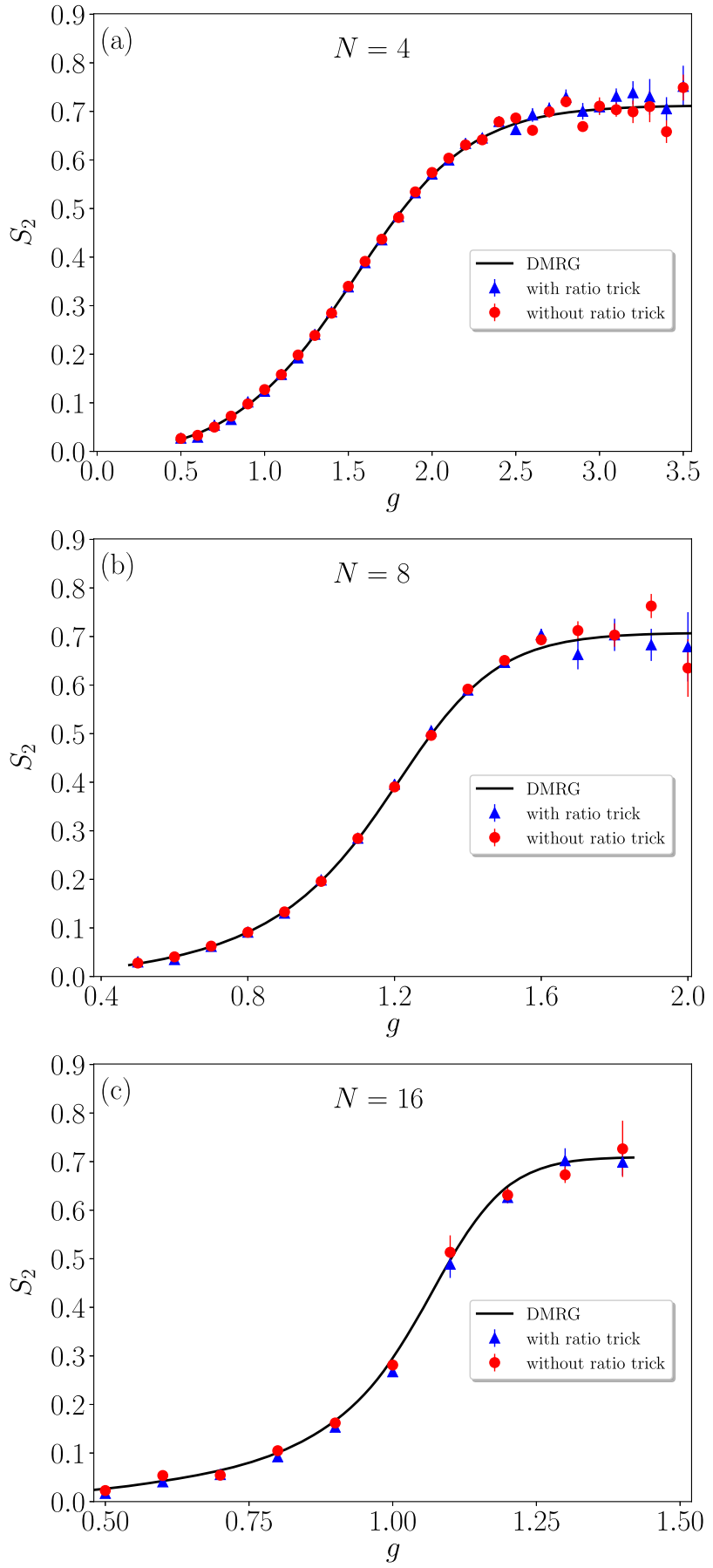

FIG. 6. Comparison of $S_{2}$ values estimated by PIGS using the extended ensemble with and without the ratio trick.

The anisotropic part of the dipole-dipole interaction in Eq. (36) breaks the $O(3)$ symmetry of the rotors, causing a "head-to-tail" alignment along the space-fixed $z$ axis. In the large $g$ and $N$ limits, we expect the ground state to have the form $(|\leftarrow \cdots \leftarrow\rangle+|\rightarrow \cdots \rightarrow\rangle) / \sqrt{2}$,
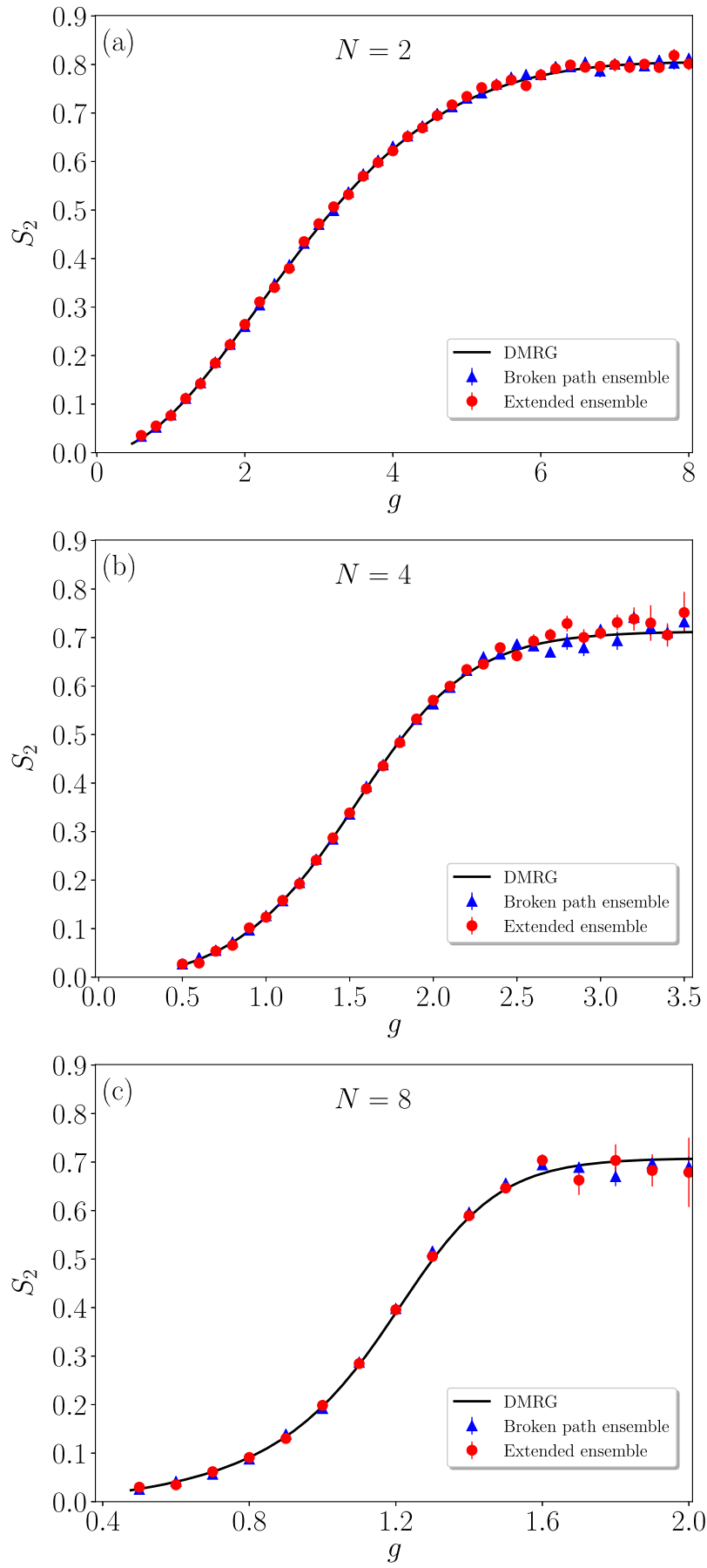

FIG. 7. Comparison of $S_{2}$ values estimated by PIGS using the broken path ensemble (without the ratio trick) and the extended ensemble (with the ratio trick).

which is known as a "cat state" due to the presence of entanglement across a macroscopically large system. In such a state, the entanglement entropy for any bipartitioning of the system is exactly $\log (2)$. Even though we have not yet simulated very large systems, we can 

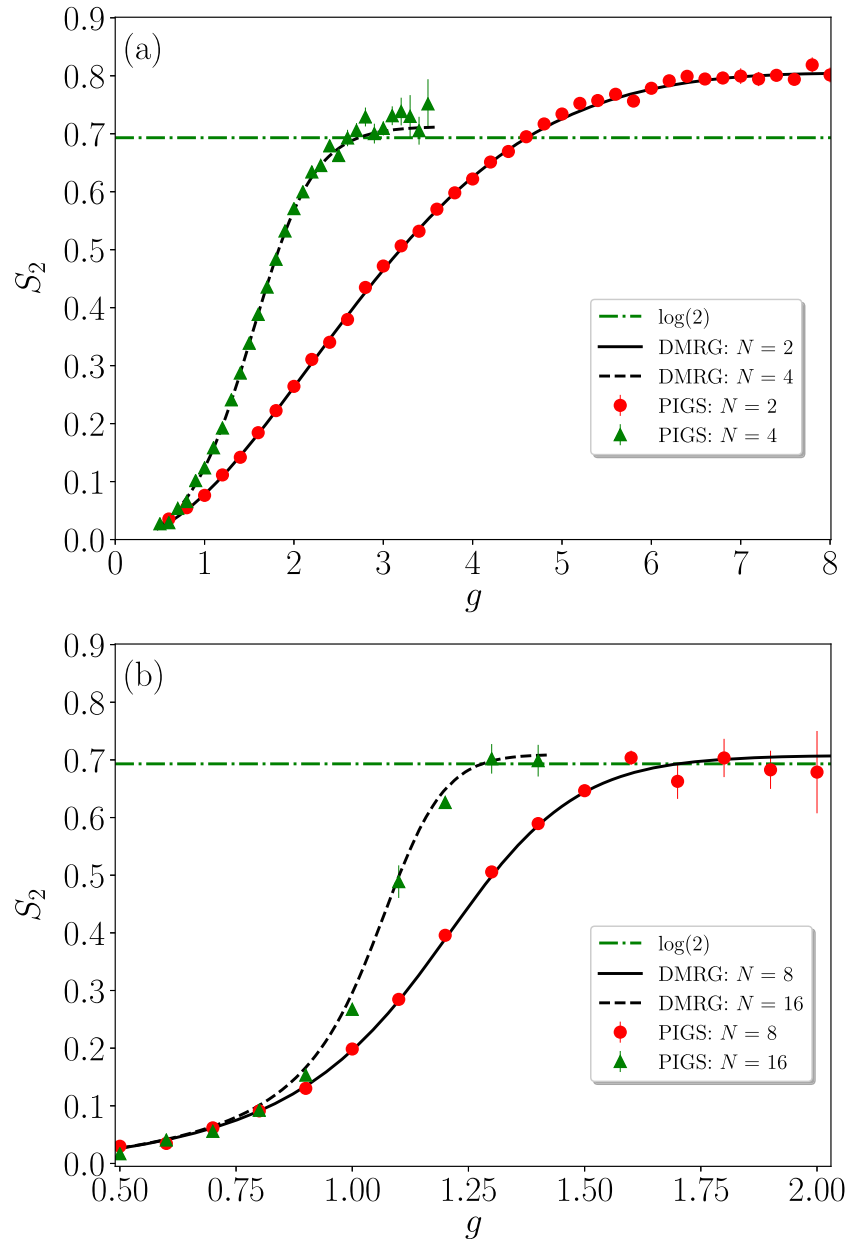

FIG. 8. $S_{2}$ values computed using PIGS simulations with the extended ensemble and ratio trick. $\log (2)$ is shown as a green dashed-dotted line.

already see the tendency of $S_{2}$ to approach a value close to $\log (2)$ when $g$ is increased.

We can gain a better understanding of the rotor alignment by observing the instantaneous values of $\cos (\theta)$ at the middle beads of all rotors in a PIGS simulation, as summarized by the histograms in Fig. 9. As the interaction strength $g$ increases, the rotors become more aligned to the $z$ axis in the space-fixed coordinates $(|\cos \theta| \rightarrow 1)$ for both $N=2$ and 8 . Although the distribution functions for $N=2$ are highly symmetric, even at very large values of $g$, an asymmetry starts to develop for $N=8$ at $g=2$. For much larger systems, where a flip of the entire rotor chain becomes extremely improbable, it is very likely that this asymmetry will give rise to non-ergodicity of the Monte Carlo sampling, which could potentially be remedied by more sophisticated spatial updates.

In this study, the most computationally expensive calculation is the estimation of $S_{2}$ for $N=16$ with an imaginary time step of $\tau=0.0033 \mathrm{~K}^{-1}$ and $P=61$ beads. Using the ratio trick algorithm expressed in Eq. (35), we run eight separate simulations for the aforementioned system, and each simulation takes $\sim 280 \mathrm{~h}$ (for 61
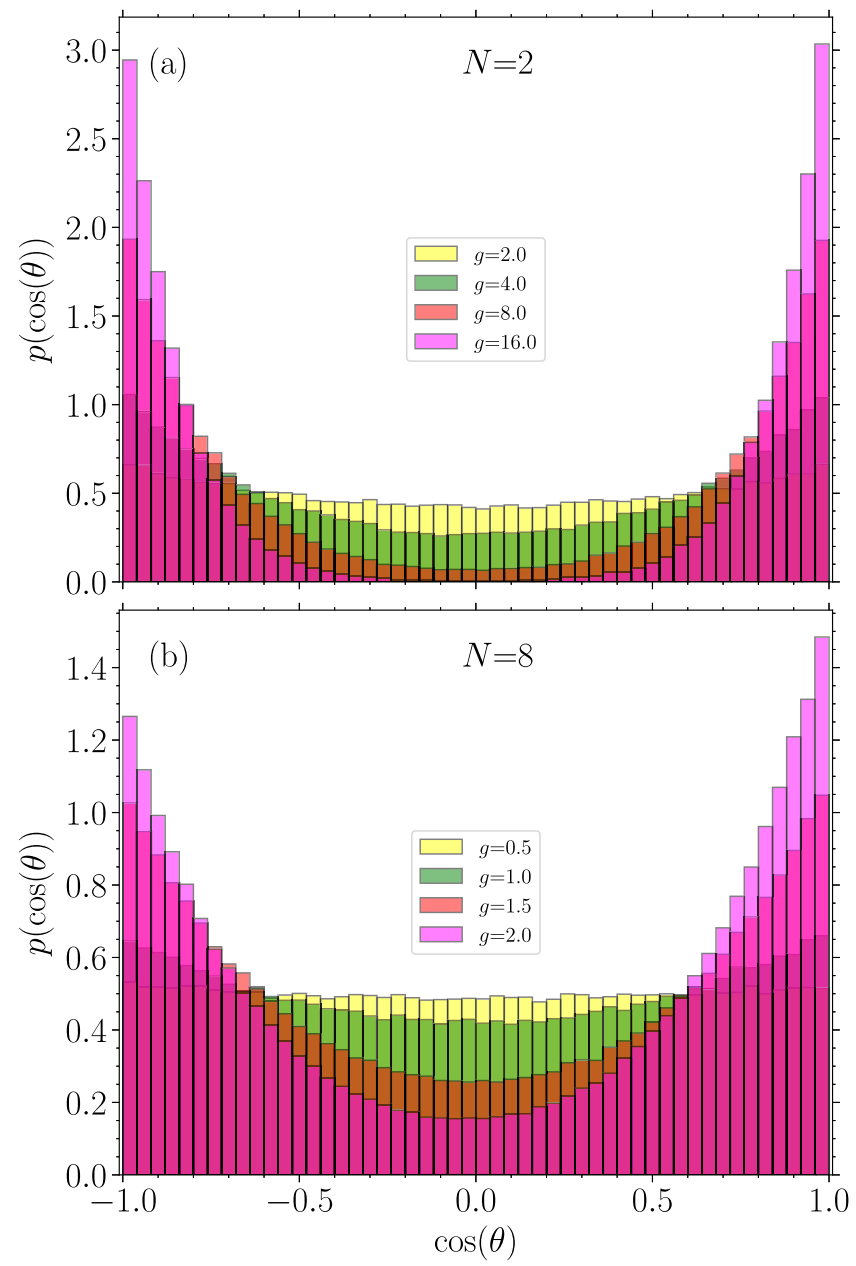

FIG. 9. Orientational probability distribution functions $p(\cos (\theta))$ for different interaction strengths $g$ at (a) $N=2$ and (b) $N=8$. $\theta$ is the angle between the dipole moment vector of a rotor and the $z$ axis in the space-fixed coordinates. The histograms are generated from extended ensemble PIGS simulations with an imaginary time step $\tau=0.0033 \mathrm{~K}^{-1}$.

$\times 10^{6}$ Monte Carlo steps) on a single core of an Intel Xeon E5-2683 $\mathrm{v} 4$ Broadwell processor, which has a base frequency of $2.1 \mathrm{GHz}$.

\section{CONCLUDING REMARKS}

We have compared the broken path ensemble and the extended ensemble (with and without the ratio trick) for PIGS based on the replica algorithm for the computation of the second Rényi entropy of a bipartite system with continuous rotational degrees of freedom. We have shown that such simulations are feasible by studying systems containing dipolar linear rotors pinned to a regular linear chain. We observe that the rotational second Rényi entropy of a symmetrically bipartite system of coupled dipoles increases with an increase in interaction strength $g$ until it reaches a constant value near $\log (2)$. We believe that this is due to the interaction potential function being minimized when the dipoles are aligned head-to-tail, 
and it reveals the role of the anisotropy of the dipole-dipole interaction in inducing orientational order. To our knowledge, this is the first PIGS estimation of entanglement entropy in a one-dimensional dipolar quantum rotor model.

The methodology that we have used can be further extended to include translational motion. This will allow the exploration of the effect of interaction strength on the second Rényi entropy of a bipartite system with translational and rotational degrees of freedom on an equal footing. This method can then be applied to dipoles with more realistic interaction potentials that accurately model the short range interaction and include higher order multipole contributions. Future work will focus on PIGS calculations of more complex molecular rotors such as asymmetric tops. ${ }^{31,32}$

It is also straightforward to extend the PIGS simulations to twoand three-dimensional lattices, something that would be challenging for the DMRG approach used as a benchmark here. Interesting PIGS results of quantum dipolar rotors have recently been obtained, ${ }^{33}$ and it will be interesting to study the scaling of $S_{2}$ in higher dimensions for molecular rotors. We note that the higher order actions and specialized trial functions used in Ref. 33 are of great interest and will be the subject of future work.

In this work, we have focused on a one-dimensional system that we expect to display an area-law scaling of entanglement, and consequently, the entanglement entropy should saturate to a constant with an increase in the subsystem size. In higher dimensions, the entanglement entropy of area-law systems will grow (linearly or faster) with the subsystem size, and thus, the statistical error of the entropy estimator will diverge exponentially with the size of the subsystem. Hence, although we did not find a clear computational advantage of the ratio trick and extended ensemble in this work, we expect that these methods will provide an algorithmic advantage in higher dimensional systems.

While the immediate aim of our work is to present our development of a PIGS approach for the estimation of the second Rényi entropy of interacting rotors, we believe that this methodological advance will enable the characterization of quantum phase transitions in rotor systems. As a longer-term objective, we hope that this knowledge will open the door to the characterization and implementation of quantum information devices based on trapped polar molecules $^{34}$ and their rotational degrees of freedom. ${ }^{35-38}$

\section{ACKNOWLEDGMENTS}

The authors acknowledge the Natural Sciences and Engineering Research Council (NSERC) of Canada (Grant No. RGPIN-201604403), the Ontario Ministry of Research and Innovation (MRI), the Canada Research Chair program (Grant No. 950-231024), the Canada Foundation for Innovation (CFI) (Project No. 35232), Compute Canada, and the Canada First Research Excellence Fund (CFREF). The authors thank Dr. Tom Halverson and Dr. Yulia Kalugina for fruitful discussions.

\section{APPENDIX: JUSTIFICATION OF QUADRATIC $\tau$ FIT FOR $S_{2}$}

For sufficiently small systems, it is possible to compute the second Rényi entropy $S_{2}$ with the inclusion of Trotter factorization error due to finite $\tau$ without performing Monte Carlo sampling. We

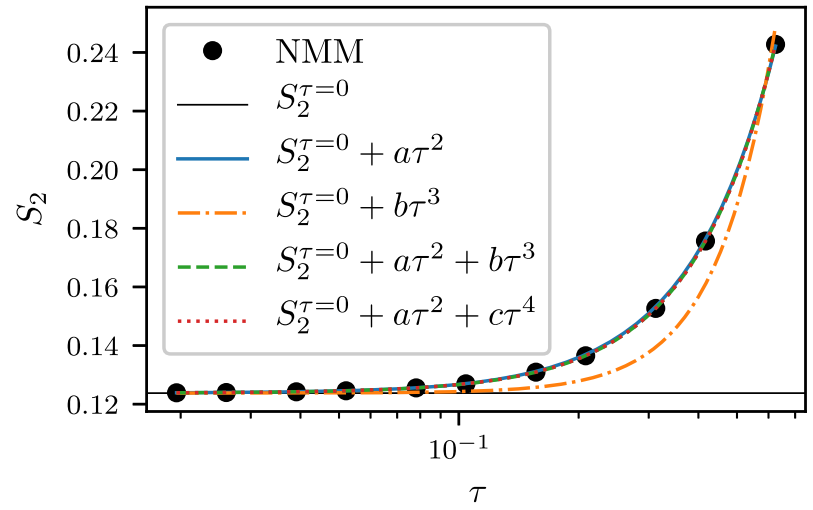

FIG. 10. Least squares fits of $S_{2}(\tau)$ values obtained from numerical matrix multiplication (NMM) for $N=4$ and $g=1$ (the specific values of $\beta$ and $\tau$ are arbitrary in this example). The exact value $S_{2}^{\tau=0}$ is specified directly, while the coefficients $a$, $b$, and $c$ are determined by fitting.

do this for $N=4$ and $g=1$ using numerical matrix multiplication (NMM), where the path is computed from a product of explicitly constructed matrices.

The data are shown in Fig. 10, along with several least squares fits. It is clear from this example that the leading contribution to the Trotter factorization error in $S_{2}$ is quadratic in $\tau$, so a fitting model of the form

is appropriate.

$$
S_{2}(\tau)=S_{2}^{\tau=0}+a \tau^{2}
$$

\section{REFERENCES}

${ }^{1}$ I. Bengtsson and K. Życzkowski, Geometry of Quantum States: An Introduction to Quantum Entanglement, 1st ed. (Cambridge University Press, 2006), p. 301.

${ }^{2}$ A. Rényi, Proceedings of the Fourth Berkeley Symposium on Mathematical Statistics and Probability, Contributions to the Theory of Statistics Vol. 1 (University of California Press, 1961), pp. 547-561.

${ }^{3}$ A. Kitaev and J. Preskill, Phys. Rev. Lett. 96, 110404 (2006).

${ }^{4}$ M. Levin and X.-G. Wen, Phys. Rev. Lett. 96, 110405 (2006).

${ }^{\mathbf{5}}$ H.-C. Jiang, Z. Wang, and L. Balents, Nat. Phys. 8, 902 (2012).

${ }^{6}$ M. B. Hastings, I. González, A. B. Kallin, and R. G. Melko, Phys. Rev. Lett. 104, 157201 (2010).

${ }^{7}$ A. B. Kallin, M. B. Hastings, R. G. Melko, and R. R. P. Singh, Phys. Rev. B 84, 165134 (2011).

${ }^{8}$ S. Humeniuk and T. Roscilde, Phys. Rev. B 86, 235116 (2012).

${ }^{9}$ C. M. Herdman, S. Inglis, P.-N. Roy, R. Melko, and A. Del Maestro, Phys. Rev. E 90, 013308 (2014).

${ }^{10}$ C. M. Herdman, A. Rommal, and A. Del Maestro, Phys. Rev. B 89, 224502 (2014).

${ }^{11}$ C. M. Herdman, P.-N. Roy, R. G. Melko, and A. Del Maestro, Phys. Rev. B 89, 140501 (2014).

${ }^{12}$ C. M. Herdman, P.-N. Roy, R. G. Melko, and A. D. Maestro, Nat. Phys. 13, 556 (2017).

${ }^{13}$ D. M. Ceperley, Rev. Mod. Phys. 67, 279 (1995).

${ }^{14}$ A. Sarsa, K. E. Schmidt, and W. R. Magro, J. Chem. Phys. 113, 1366 (2000).

${ }^{15}$ T. Halverson, D. Iouchtchenko, and P.-N. Roy, J. Chem. Phys. 148, 074112 (2018).

${ }^{16}$ D. Iouchtchenko and P.-N. Roy, J. Chem. Phys. 148, 134115 (2018).

${ }^{17}$ H. F. Trotter, Proc. Am. Math. Soc. 10, 545 (1959).

${ }^{18}$ D. Marx and M. H. Müser, J. Phys.: Condens. Matter 11, R117 (1999). 
${ }^{19}$ D. Iouchtchenko, M.S. thesis, University of Waterloo, 2015.

${ }^{20}$ D. Iouchtchenko and P.-N. Roy, "Recent progress in quantum Monte Carlo," ACS Symp. Ser. 1234, 145 (2016).

${ }^{21}$ M. Boninsegni, N. V. Prokof'ev, and B. V. Svistunov, Phys. Rev. E 74, 036701 (2006).

${ }^{22}$ In practice, we observe that attempting this connectivity update after every spatial update (that is, a batch size of 1 ) is reasonable.

${ }^{23}$ N. Metropolis, A. W. Rosenbluth, M. N. Rosenbluth, A. H. Teller, and E. Teller, J. Chem. Phys. 21, 1087 (1953).

${ }^{24}$ S. Inglis and R. G. Melko, New J. Phys. 15, 073048 (2013).

${ }^{25}$ B. P. Abolins, R. E. Zillich, and K. B. Whaley, J. Low Temp. Phys. 165, 249 (2011).

${ }^{26}$ B. P. Abolins, R. E. Zillich, and K. B. Whaley, J. Low Temp. Phys. 170, 131 (2013).

${ }^{27}$ A. Krachmalnicoff, R. Bounds, S. Mamone, S. Alom, M. Concistrè, B. Meier, K. Kouřil, M. E. Light, M. R. Johnson, S. Rols, A. J. Horsewill, A. Shugai, U. Nagel, T. Rõõm, M. Carravetta, M. H. Levitt, and R. J. Whitby, Nat. Chem. 8, 953 (2016).
${ }^{28}$ Y. N. Kalugina and P.-N. Roy, J. Chem. Phys. 147, 244303 (2017).

${ }^{29}$ T. Zeng, N. Blinov, G. Guillon, H. Li, K. P. Bishop, and P.-N. Roy, Comput. Phys. Commun. 204, 170 (2016).

${ }^{30}$ T. Zeng and P.-N. Roy, Rep. Prog. Phys. 77, 046601 (2014).

${ }^{31}$ T. Zeng, H. Li, and P.-N. Roy, J. Phys. Chem. Lett. 4, 18 (2013).

${ }^{32}$ T. Zeng, G. Guillon, J. T. Cantin, and P.-N. Roy, J. Phys. Chem. Lett. 4, 2391 (2013).

${ }^{33}$ B. P. Abolins, R. E. Zillich, and K. B. Whaley, J. Chem. Phys. 148, 102338 (2018).

${ }^{34}$ D. DeMille, Phys. Rev. Lett. 88, 067901 (2002).

${ }^{35}$ C. P. Koch, M. Lemeshko, and D. Sugny, Rev. Mod. Phys. 91, 035005 (2019).

${ }^{36}$ V. V. Albert, J. P. Covey, and J. Preskill, arXiv:1911.00099 (2019).

${ }^{37}$ M. Hughes, M. D. Frye, R. Sawant, G. Bhole, J. A. Jones, S. L. Cornish, M. Tarbutt, J. M. Hutson, D. Jaksch, and J. Mur-Petit, arXiv:1912.09419 (2019).

${ }^{38}$ J. M. Hutson, J. Aldegunde, J. Blackmore, D. Jaksch, M. Tarbutt, P. Gregory, S. Cornish, R. Sawant, and J. Petit, New J. Phys. 22, 013027 (2020). 